PHYSICAL REVIEW C 72, 059903(E) (2005)

\title{
Publisher's Note: Momentum-space description of three-nucleon breakup reactions including the Coulomb interaction [Phys. Rev. C 72, 054004 (2005)]
}

\author{
A. Deltuva, A. C. Fonseca, and P. U. Sauer \\ (Received 21 November 2005; published 30 November 2005)
}

DOI: 10.1103/PhysRevC.72.059903

PACS number(s): 21.30.-x, 21.45.+v, 24.70.+s, 25.10.+s, 99.10.Fg

This paper was published online on 9 November 2005 with a typographical error on page 1 . On page 1, in the first paragraph of the Introduction, the citations in the sixth line should read as "...very few attempts [6-8] that ...." The text has been corrected as of 22 November 2005. The text is correct in the printed version of the journal. 